\section{Systèmes endocrines et métabolisme}

\title{
Thermorégulation, obésité et système ortho-sympathique: invalidation des gènes Ucp1 et Dbh
}

L'étude des mécanismes permettant à des petits mammifères de lutter contre le froid, a mis en évidence le rôle de leur tissu adipeux brun. Ce tissu est abondant chez les mammifères de petite taille, les mammifères nouveau-nés, ainsi que chez les animaux hibernants. Les adipocytes bruns sont des cellules spécialisées dans la production de chaleur due à la présence de nombreuses mitochondries dont la respiration n'est pas couplée à la phosphorylation de l'ADP. Le découplage respiratoire est réalisé par UCPl (uncoupling protein ou protéine découplante), une protéine spécifique des adipocytes bruns et agissant en tant que protonophore dans la membrane interne mitochondriale [1]. Par ailleurs, il est connu que le système orthosympathique est impliqué dans la thermogenèse induite par l'exposition au froid. En fait, l'activité et la croissance du tissu adipeux brun sont contrôlées par le système nerveux ortho-sympathique, les adipocytes bruns étant directement innervés par des fibres orthosympathiques. Lors de l'exposition au froid, à la naissance, ou au moment du réveil dans le cas d'un hibernant, les fibres sympathiques libèrent de la noradrénaline qui se lie à des récepteurs adrénergiques (en particulier les récepteurs adrénergiques $\beta 3$, [2]) présents à la surface des adipocytes bruns. Lors de l'activation des adipocytes bruns, les acides gras libres provenant de la lipolyse activent l'UCP; la respiration est alors découplée, et de la chaleur est produite.

Deux publications récentes décrivent, l'une, les effets de l'invalidation du gène Ucpl [3], l'autre les effets de l'invalidation du gène $D b h$ codant pour une enzyme de la voie de biosynthèse de la noradrénaline et de l'adrénaline [4]. Les animaux $U c p^{-/}$- sont très sensibles au froid et ne peuvent maintenir leur température corporelle à un niveau élevé s'ils sont exposés à $4{ }^{\circ} \mathrm{C}$. Par ailleurs, leurs adipocytes bruns accumulent beaucoup de triglycérides et ils ne peuvent plus accroître leur consommation d'oxygène s'ils reçoivent un agoniste des récepteurs adrénergiques $\beta 3$. Ces résultats prouvent le rôle biologique essentiel du gène Ucpl dans la thermogenèse induite par le froid. Ils confirment aussi le rôle de la protéine UCP1 dans la stimulation de l'oxydation des graisses, ainsi que l'importance du tissu adipeux brun dans l'augmentation de la consommation d'oxygène observée lors de l'administration de catécholamines. Cependant, d'autres travaux ayant montré que le tissu adipeux brun pouvait contribuer à dissiper une fraction de l'énergie des aliments [5], les auteurs de l'invalidation du gène Ucpl s'attendaient à observer une obésité chez leurs animaux, ce qui ne fut pas le cas. Ils ont fait l'hypothèse d'un mécanisme compensateur s'opposant au stockage des graisses. En fait, ce mécanisme de compensation semble exister, puisque chez les animaux $U c p^{-} /^{-}$, une surexpression du gène Ucp2 est observée uniquement dans le tissu adipeux brun. La protéine UCP2 est une protéine homologue d'UCP1, récemment découverte, capable de découpler la respiration et participant à la dépense énergétique $(\mathrm{m} / \mathrm{s}$ $n^{\circ} 4$, vol. 13, p. 607).
L'invalidation du gène $D b h$ (codant pour la dopamine $\beta$-hydroxylase) entraîne la mort in utero des animaux, mais l'addition de dihydroxyphénylsérine à l'eau de boisson des souris gestantes permet la survie des souriceaux [6]. A la température ambiante de $4^{\circ} \mathrm{C}$, la température corporelle des souris $\mathrm{Dbh}^{-} /^{-}$baisse de $13^{\circ} \mathrm{C}$ au cours de la première heure [4]. Ces animaux sont incapables de lutter contre le froid en accroissant leur thermogenèse. Cela peut s'expliquer par l'absence de stimulation orthosympathique du tissu adipeux brun et par la non activation d'UCP1. Mais les auteurs ont montré, qu'à l'absence d'activation du tissu adipeux brun, s'ajoutent des défauts de diminution des pertes de chaleur: les souris $\mathrm{Dbh}^{-} /^{-}$sont incapables d'effectuer une vasoconstriction périphérique, et présentent une déficience de piloérection. Étant donné le rôle lipolytique des catécholamines, on attendait une obésité, d'autant plus que les souris $D b h^{-} /$ sont hyperphagiques. En fait, ces souris ne sont pas obèses car elles ont un métabolisme de base accru. Ni le frisson, ni une hyperthyroïdie, ni une surproduction d'UCP2 (comme cela est observé chez les souris $U c p^{-} /^{-}$), ne semblent expliquer l'augmentation du métabolisme de base des souris $D b h^{-} /$. La destruction du gène de la dopamine $\beta$-hydroxylase confirme donc l'importance des catécholamines dans les mécanismès thermorégulateurs, et démontre l'existence de mécanismes inconnus de contrôle du métabolisme de base.
D.R. 
1. Ricquier D. Thermogenèse et obésité : mécanismes moléculaires. Med Sci 1985 ; 1 : 147-53.

2. Emorine I, Strosberg D. Structure et fonction du récepteur $\beta$-3 adrénergique. Med Sci 1993 ; 9 : 1228-35.
3. Enerbāck S, Jacobsson A, Simpson EM, Guerra C, Yamashita H, Harper ME, Kozak LP. Mice lacking mitochondrial uncoupling protein are coldsensitive but not obese. Nature $1997 ; 387$ : 90-4. 4. Thomas SA, Palmiter RD. Thermoregulatory and metabolic phenotypes of mice lacking nora- drenaline and adrenaline. Nature 1997; 387 : 94-7. 5. Seydoux J. Défaut de thermogenèse et obésité Med Sci 1987 ; 3 : 387-93.

6. Thomas SA, Matsumoto AM, Palmiter RD Noradrenaline is essential for mouse fetal development. Nature 1995 ; 374 : 643-6.
La molécule inflammatoire (MIF) et l'insuline : une promiscuité d'intérêt! le MIF (macrophage migration inhibitory factor) fait son entrée aujourd'hui dans le domaine du métabolisme glucidique. L'identification de la protéine dans la cellule $\beta$ pancréatique et son effet stimulant sur la sécrétion d'insuline [1] enrichissent encore la panoplie des multiples fonctions de MIF. Produit par les lymphocytes $\mathrm{T}$, les macrophages et l'hypophyse, le MIF est un médiateur important dans les processus pro-inflammatoires et la réponse au choc septique, et contrecarre les effects immunosuppresseurs et anti-inflammatoires des glucocorticoïdes. Une étude germano-helvético-américaine démontre, chez la souris, la présence de la protéine dans les cellules $\beta$ pancréatiques, et sa co-localisation avec l'insuline dans des granules de sécrétion. Dans des îlots de rat, comme dans la lignée cellulaire INS-1 insulino-sécrétrice, le glucose induit une augmentation (de 3 et 4 fois) de la quantité basale d'ARNm de MIF. Le glucose étant activateur de la production de MIF, et de la sécrétion d'insuline, l'hypothèse d'un effet régulateur de MIF sur la sécrétion d'insuline était séduisante. Cet effet a été effectivement mis en évidence à l'aide d'anticorps anti-MIF : ceux-ci inhibent la sécrétion d'insuline par des îlots de rat périfusés en milieu glucosé, phénomène réversible après retrait de l'anticorps. Confirmant cette observation, l'ajout de MIF recombinant à la préparation augmente la sécrétion insulinique des îlots induite par le glucose. Enfin, dans des cellules INS-1 transfectées avec un vecteur d'expression plasmidique $M I F$ anti- sens, une nette diminution de la sécrétion d'insuline est observée. Ces résultats indiquent que MIF, produit de sécrétion de la cellule $\beta$, fonctionne comme un régulateur autocrine de la sécrétion d'insuline. Outre son rôle dans la réponse à l'inflammation, l'infection et le stress, on peut penser que, dans des conditions inflammatoires chroniques, une concentration importante de MIF circulant potentialise la sécrétion d'insuline pour préserver l'intégrité métabolique de l'hôte et assurer sa survie.

[1. Waeber G, et al. Proc Natl Acad Sci USA 1997 ; 94 : 4782-7.]

NFl et voie de l'AMP cyclique... au moins chez la drosophile. $N F 1$ est le gène impliqué dans la neurofibromatose de type 1 . Il code pour une protéine GAP (GTPase-activating protein) $\left(\mathrm{m} / \mathrm{s} n^{\circ} 1\right.$, vol. 8, p. 91). De nombreux arguments indiquent qu'une hyperactivité des voies de transmission des signaux passant par l'activation de Ras, liée aux défauts de désactivation de Ras-GTP en Ras-GDP, est impliquée dans la physiopathologie des neurinomes multiples caractérisant la neurofibromatose de type 1 [1]. L'équipe d'André Bernards (Charlestown, MA, USA), en collaboration avec celle de J.F. Gusella, rapporte maintenant que, chez la drosophile, le gène $N f 1$ semblait plus impliqué dans la transmission du signal passant par la protéine kinase A (PKA) stimulée par l'AMP cyclique que par celles de Ras [2, $3]$. Des drosophiles déficientes en NF1 sont viables mais ont une petite taille. Cela contraste avec le phénotype des souris $\mathrm{Nfl}^{-/-}$qui meurent durant le développement embryonnaire [1]. De manière encore plus surprenante, le phénotype des drosophiles homozygotes pour le déficit en NF1 est restauré par transfert d'un gène codant pour la sous-unité catalytique de la PKA [2]. De belles expériences de génétique montrent que le signal passant par un neuropeptide PACAP38, connu pour activer la synthèse de l'AMP cyclique (pituitary adenylyl cyclase-activating polypeptide), est inhibé chez les mouches déficientes en NF1. Cette inhibition semble être due à un blocage chez ces mouches de l'activation de l'adénylyl cyclase, codée par le gène rutabaga $\left(\mathrm{m} / \mathrm{s} n^{\circ} 8\right.$, vol. 11 , p. 1175). L'action du peptide PACAP38 sur un courant potassium, qui exige à la fois la stimulation de la voie AMP cyclique et de la voie Ras-Raf, est restaurée par des agents pharmacologiques augmentant la concentration intracellulaire de l'AMP cyclique. Ces résultats obtenus chez la drosophile invitent maintenant à reposer la question du mode d'action de NF1 chez les mammifères. Chez ces derniers, outre la régulation négative de Ras, NFl agit-il également comme un stimulateur de l'adénylyl cyclase et, ainsi, comme un régulateur positif du signal relayé par l'AMP cyclique.

[1. Henry I. Med Sci 1995; 11 : 938.]

[2. The I, et al. Science 1997; 276 : 791-4.]

[3. Guo HF, et al. Science 1997 ; 276 : 795-8.] 